\title{
Graded Manifolds, Supermanifolds and Infinite-Dimensional Grassmann Algebras
}

\author{
Alice Rogers ${ }^{\star}$ \\ Department of Mathematics, King's College, Strand, London WC2, England
}

\begin{abstract}
A new approach to superdifferentiable functions of Grassmann variables is developed, which avoids ambiguities in odd derivatives. This is used to give an improved definition of supermanifold over a finite-dimensional Grassmann algebra. A natural embedding of super-manifolds over Grassmann algebras with increasing number $(L)$ of generators is developed, and thus a limit as $L$ tends to infinity is possible. A correspondence between graded manifolds and supermanifolds is constructed, extending results of [5] and [8].
\end{abstract}

\section{Introduction}

In recent years much conventional differential geometry has been extended to include anticommuting variables; objects in this extended field of study, such as supermanifolds and super Lie groups, are distinguishable by the prefix "super" which derives from the same prefix in supersymmetry, the fermi-base symmetry which is under such intense study by elementary particle (and string) physicists.

Historically, the consideration of supermanifolds has a dual origin. The earliest work, due to Berezin and Leites [1] and Kostant [2] arose from the study of the mathematics of fermi field quantisation; their approach was sheaf theoretic, extending the sheaf of $C^{\infty}$ functions on a manifold, rather than the manifold itself. (From now on, supermanifolds defined in this manner will, following Kostant, be described as graded manifolds.) The second, more geometric, approach grew directly from the physicist's superspace [3] as a space with points labelled by even elements $\left(x^{\mu}\right)$ and odd elements $\left(\theta^{\alpha}\right)$ of a Grassmann algebra; a supermanifold is a topological space with local coordinates $\left(x^{\mu} ; \theta^{\alpha}\right)$ of this nature $[4,5]$. A good recent review is by Batchelor [6].

The present paper has three aims: one is to improve the definition of a "superdifferentiable" function on a supermanifold, another is to relate supermanifolds defined over Grassmann algebras with different numbers of generators, and hence give a new approach to handling Grassmann algebras with unlimited numbers of generators. The final aim is to complete the analysis of the corre- 
spondence between graded manifolds and supermanifolds. These three aims will now be described in more detail.

One slightly unsatisfactory aspect of supermanifold theory has been the difficulty in deciding how many generators one's Grassmann algebra should have. For physical applications it seems desirable to have an unlimited number of generators, to avoid non-miraculous cancellations (for instance, when multiplying together Green's functions at different points in superspace). One way of employing an infinite-dimensional Grassmann algebra (by imposing an $l^{1}$ norm) was introduced by the author in [5]; subsequently Jadzyck and Pilch [7] defined general conditions for an infinite-dimensional Banach Grassmann algebra appropriate to supermanifolds, but the algebra constructed in [5] remains the only example of such an algebra so far constructed. The method of [5] is analytically quite satisfactory, but has the drawback that not all $C^{\infty}$ functions of the reals can be extended to the infinite-dimensional Grassmann algebra in the natural way described below (Eqs. 2.11 and 2.13) for finite-dimensional algebras.

In this paper an alternative approach to having an infinite number of generators is described; the method arose naturally in the course of considering two relatively trivial but rather niggling problems in supermanifold theory. One is the fact that, if one has a finite number of Grassmann generators, odd derivatives are not welldefined (as is explained in Sect. 2); the other is that supermanifolds over Grassmann algebras with different numbers of generators are treated as different categories, and clearly the relation between the categories needs investigation; (for a restricted class of supermanifold this was done by Batchelor [8].)

In Sect. 2, a new approach to the idea of superdifferentiable function, giving welldefined odd derivatives, is described; loosely, the idea is to keep a few spare Grassmann generators up one's sleeve to avoid unwanted cancellations.

In Sect. 3 it is shown how a supermanifold over a Grassmann algebra with $L$ generators (where $L$ is some positive integer) can always be imbedded naturally into one over a Grassmann algebra with $L+1$ generators. For a physicist wishing to do practical (so to speak) calculations, this means that whenever cancellations occur because of a shortage of Grassmann generators, one can simply move to a space with more generators; mathematically, one can take a direct limit.

Returning to the sheaf-theoretic approach $[1,2]$, an $(m, n)$ dimensional graded manifold is a pair $(X, A)$ where $X$ is an $m$-dimensional real manifold, and $A$ is a sheaf of graded commutative algebras over $X$ which locally decomposes in the following manner: there is an open cover $\left\{U_{\alpha} \mid \alpha \in \Lambda\right\}$ of $X$ such that for each $\alpha$ in $\Lambda$

$$
A\left(U_{\alpha}\right) \simeq C\left(U_{\alpha}\right) \otimes \Lambda\left(\mathbb{R}^{n}\right),
$$

where $\Lambda\left(\mathbb{R}^{n}\right)$ denotes the exterior algebra over $\mathbb{R}^{n}$, and there is an algebra isomorphism

$$
c: C\left(U_{\alpha}\right) \rightarrow C^{\infty}\left(U_{\alpha}\right) \text {. }
$$

Thus a typical element $f$ of $A\left(U_{\alpha}\right)$ may be expanded as

$$
f=c^{-1}\left(f_{\phi}\right)+\sum_{j=1}^{n} c^{-1}\left(f_{(j)}\right) \theta^{j}+\sum_{i<j=1}^{n} c^{-1}\left(f_{(i j)}\right) \theta^{i} \theta^{j}+\cdots,
$$


where the $\theta^{j}$ are generators of $\Lambda\left(\mathbb{R}^{n}\right)$, and the $f_{\phi}, f_{(j)}, f_{(i j)}$ and so on are elements of $C^{\infty}\left(U_{\alpha}\right)$. (Complex graded manifolds may be defined similarly.)

It has been proved $[5,8]$ that in the geometric approach to supermanifolds these sheaves of abstract algebras can be realised as actual function algebras, (but to do this one has to use a class of functions on a supermanifold which is much more restricted than the full class of superdifferentiable functions.) At first sight the sheaftheoretic approach seems more economical (and hence elegant) because, although it involves the Grassmann algebra $\Lambda\left(\mathbb{R}^{n}\right)$ it does not rely on an auxiliary Grassmann algebra where coordinates take their values. However, for applications to supersymmetry, the expansion (1.2) is not of the correct form to correspond to a superfield, because the coefficients $f_{\phi}, f_{(j)}$ etc. are all commuting. This may easily be remedied by replacing (1.1) by

$$
A\left(U_{\alpha}\right) \simeq C\left(U_{\alpha}\right) \otimes \Lambda\left(\mathbb{R}^{n}\right) \otimes \Lambda\left(\mathbb{R}^{L}\right),
$$

where $L$ is an integer greater than $n$. But now the virtue of the sheaf-theoretic approach, that it avoids using an auxiliary Grassmann algebra, is lost. In Sect. 4 the sheaves of algebras satisfying (1.4) are shown precisely to correspond to the superdifferentiable functions defined in Sect.2, and thus the correspondence between supermanifolds and graded manifolds is made complete. Section 5 contains a brief summary.

\section{Superdifferentiable Functions of Grassmann Variables}

In this section, attention is restricted to Grassmann algebras with a finite number of generators; for simplicity, only algebras over the reals will be considered. For each positive integer $L, B_{L}$ will denote the algebra over the reals with generators $1^{(L)}$, $\beta_{1}^{(L)}, \ldots, \beta_{L}^{(L)}$, and relations

$$
\begin{array}{ll}
1^{(L)} \beta_{i}^{(L)}=\beta_{i}^{(L)} 1^{(L)}=\beta_{i}^{(L)} & i=1, \ldots, L, \\
\beta_{i}^{(L)} \beta_{j}^{(L)}=-\beta_{j}^{(L)} \beta_{i}^{(L)} & i, j=1, \ldots, L .
\end{array}
$$

A useful notation (due to Kostant [2]) is to let $M_{L}$ denote the set of finite sequences of positive integers $\mu=\left(\mu_{1}, \ldots, \mu_{k}\right)$ with $1 \leqq \mu_{1}<\cdots<\mu_{k} \leqq L . M_{L}$ includes the sequence with no elements, denoted $\phi$. Then, if for each $\mu$ in $M_{L}$,

and

$$
\beta_{\mu}^{(L)}:=\beta_{\mu_{1}}^{(L)} \cdots \beta_{\mu_{k}}^{(L)},
$$

$$
\beta_{\phi}^{(L)}:=1^{(L)},
$$

a typical element $b$ of $B_{L}$ may be expressed as

$$
b=\sum_{\mu \in M_{L}} b^{\mu} \beta_{\mu}^{(L)},
$$

where the coefficient $b^{\mu}$ are real numbers. A useful map is the augmentation map (or body map, in DeWitt's terminology [4]) $\varepsilon^{(L)}: B_{L} \rightarrow \mathbb{R}$ defined by

$$
\varepsilon^{(L)}(b)=b_{\phi} .
$$


With the norm on $B_{L}$ defined by

$$
\|b\|:=\sum_{\mu \in M_{L}}\left|b^{\mu}\right|
$$

$B_{L}$ is a Banach algebra.

An obvious point, basic to the new construction in this paper, is that if $L^{\prime}$ is also a positive integer, with $L \geqq L^{\prime}$, then there is a natural injection $l_{L^{\prime}, L}: B_{L^{\prime}} \rightarrow B_{L}$, which is the unique algebra homomorphism satisfying

$$
l_{L^{\prime}, L}\left(\beta_{i}^{\left(L^{\prime}\right)}\right)=\beta_{i}^{(L)} \quad i=1, \ldots, L, \quad l_{L^{\prime}, L}\left(1^{\left(L^{\prime}\right)}\right)=1^{(L)} .
$$

$B_{L}$ naturally has a $B_{L^{\prime}}$ module structure with, given $a \in B_{L^{\prime}}$ and $b \in B_{L}$,

$$
a b:=l_{L^{\prime}, L}(a) b .
$$

In the case of an $(m, n)$-dimensional supermanifold, the space where coordinates take their values is the cartesian product of $m$ copies of the even part and $n$ copies of the odd part of $B_{L}$. This space will be called $B_{L}^{m, n}$; a typical element is written $\left(x^{1}, \ldots, x^{m} ; \theta^{1}, \ldots, \theta^{n}\right)$ or simply $(x ; \theta)$.

A useful map is

$$
\varepsilon_{m, n}^{(L)}: B_{L}^{m, n} \rightarrow \mathbb{R}^{m}
$$

with

$$
\varepsilon_{m, n}^{(L)}\left(x^{1}, \ldots, x^{m} ; \theta^{1}, \ldots, \theta^{n}\right):=\left(\varepsilon^{(L)}\left(x^{1}\right), \ldots, \varepsilon^{(L)}\left(x^{m}\right)\right) .
$$

A key ingredient in the theory of supermanifolds is the notion of a "superdifferentiable" function $f: B_{L}^{m, n} \rightarrow B_{L}$. In [5], two types of super-differentiable functions (called $G^{\infty}$ and $H^{\infty}$ ) were described. In this section a new class of function, intermediate between these two, called $G H^{\infty}$ will be described. $G H^{\infty}$ functions are sufficiently general to be the appropriate mathematical form for the physicist's superfield (unlike $H^{\infty}$ functions), but avoid the difficulty (which occurs with $G^{\infty}$ functions) of having odd derivatives which are not quite well defined.

To describe the class of $G H^{\infty}$ functions, a method for continuing the domain of $C^{\infty}$ functions of $\mathbb{R}^{m}$ to include even Grassmann elements is required.

Definition 2.1. (a) Suppose $U \subset \mathbb{R}^{m}$ is open. As usual, let $C^{\infty}(U, \mathbb{R})$ denote the algebra of real-valued $C^{\infty}$ functions on $U$. Also, for any two topological spaces $A$ and $B$, let $A^{B}$ denote the set of continuous functions of $A$ into $B$. Then the map

$$
Z_{0, L}: C^{\infty}(U, \mathbb{R}) \rightarrow\left[\varepsilon_{m, 0}^{(L)-1}(U)\right]^{B_{L}}
$$

is defined by

$$
\begin{aligned}
Z_{0 . L}(f)\left(x^{1}, \ldots, x^{m}\right):= & \sum_{i_{1}=0 \ldots i_{m}=0}^{L} \frac{1}{i_{1} ! \cdots i_{m} !} \\
& \cdot\left(\partial_{1}^{i_{1}} \cdots \partial_{m}^{i_{m}} f\left(\varepsilon\left(x^{1}\right), \ldots, \varepsilon\left(x^{m}\right)\right)\right) \times s\left(x^{1}\right)^{i_{1}} \cdots s\left(x^{m}\right)^{i_{m}},
\end{aligned}
$$

where

$$
s\left(x^{i}\right)=x^{i}-\varepsilon\left(x^{i}\right) 1, \quad i=1, \ldots, m .
$$


(b) Suppose $L^{\prime}$ is a positive integer with $L^{\prime} \leqq L$. Let $C^{\infty}\left(U, B_{L^{\prime}}\right)$ denote the $B_{L^{\prime}}$ module of $C^{\infty}$ functions of $U$ into $B_{L^{\prime}}$; (recall that $B_{L^{\prime}}$ is a Banach algebra, and hence a fortiori a Banach space.) Then the map $Z_{L^{\prime}, L}: C^{\infty}\left(U, B_{L^{\prime}}\right) \rightarrow\left[\varepsilon_{m, 0}^{(L)}(U)\right]^{B_{L}}$ is defined by

$$
\begin{aligned}
Z_{L^{\prime}, L}(f)\left(x^{1}, \ldots, x^{m}\right)= & \sum_{i_{1}=0 \ldots i_{m}=0}^{L} \frac{1}{i_{1} ! \cdots i_{m} !} \\
& \cdot l_{L^{\prime}, L}\left(\partial_{1}^{i_{1}} \cdots \partial_{m}^{i_{m}} f\left(\varepsilon\left(x^{1}\right), \ldots, \varepsilon\left(x^{m}\right)\right)\right) \times s\left(x^{1}\right)^{i_{1}} \cdots s\left(x^{m}\right)^{i_{m}}
\end{aligned}
$$

The $Z$ maps preserve products and composition of functions, as can be proved by using corresponding results for Taylor series. Using these $Z$ maps the three classes of functions, $H^{\infty}, G^{\infty}$ and $G H^{\infty}$ can easily be described.

Definition 2.2. Suppose $V$ is open in $B_{L}^{m, n}$ (with respect to its usual finite-dimensional vector space topology) and that $U=\varepsilon_{m, n}^{L}(V)$. (a) Suppose $L>n . G^{\infty}(V)$ denotes the set of functions $f: V \rightarrow B_{L}$ for which there exist $f_{\mu} \in C^{\infty}\left(U, B_{L}\right)$ such that

$$
f(x ; \theta)=\sum_{\mu \in M_{n}} Z_{L, L}\left(f_{\mu}\right)(x) \theta^{\mu}
$$

Here

$$
\theta^{\mu}=\theta^{\mu_{1}} \cdots \theta^{\mu_{k}}
$$

and

$$
\theta^{\phi}=1^{L}
$$

(b) Suppose $L>n . H^{\infty}(V)$ denotes the set of functions $f: V \rightarrow B_{L}$ for which there exist $f_{\mu} \in C^{\infty}(U, \mathbb{R})$ such that

$$
f(x ; \theta)=\sum_{\mu \in M_{n}} Z_{0, L}\left(f_{\mu}\right)(x) \theta^{\mu}
$$

(c) Suppose $L>2 n$ and $L^{\prime}=\left[\frac{1}{2} L\right]$, the least integer not less than $\frac{1}{2} L . G H^{\infty}(V)$ denotes the set of functions $f: V \rightarrow B_{L}$ for which there exist $f_{\mu} \in C^{\infty}\left(U, B_{L^{\prime}}\right)$ such that

$$
f(x ; \theta)=\sum_{\mu \in M_{n}} Z_{L^{\prime}, L}\left(f_{\mu}\right)(x) \theta^{\mu} .
$$

The reader may be forgiven for finding these three definitions confusingly similar! The important difference is the range of the functions $f_{\mu}$ in each case. This is most restricted in the case of $H^{\infty}$ functions, where it is evident from the expansion (2.17) that a superfield will not have suitable component fields for applications to supersymmetry. $H^{\infty}$ functions relate closely to the sheaf-theoretic approach to supermanifolds, and are considered further in Sect. 4. At the other extreme, $G^{\infty}$ functions do correspond to the usual idea of superfield [3]; the important thing is that the coefficient functions $f_{\mu}$ can be anticommuting. However, when one attempts to develop the theory of $G^{\infty}$ functions to include differentiation with respect to odd (as well as even) variables, one finds that odd derivatives are not completely welldefined [5], and that useful rules such as the Leibnitz rule for differentiating products break down (as was first pointed out by Hess and Wieloch [9]). This in turn leads to an unsatisfactory theory of vector fields on supermanifolds. (These difficulties are of course avoided if one uses the infinite-dimensional algebra of [5]). One way round the difficulty has been considered by Boyer \& Gitler [10]; however 
they still do not achieve a completely satisfactory theory of vector fields. An alternative, more abstract, approach due to Rothstein [11] solves the problem, but at the expense of using abstract sheaves rather than actual functions. The new method developed in the present paper, using $G H^{\infty}$ functions, seems to give a simple solution; odd derivatives are well-defined (because $B_{L}$ has at least $n$ more generators than $B_{L^{\prime}}$ ), while superfields can have anticommuting coefficient functions in their expansion (2.18), because the range of $f_{\mu}$ is $B_{L^{\prime}}$ and not simply $\mathbb{R}$.

The next step is to define even and odd derivatives of $G H^{\infty}$ functions; this is similar to the standard approach for $G^{\infty}$ or $H^{\infty}$ functions.

Definition 2.3. With the notation of Definition 2.2, let $f$ be an element of $G H^{\infty}(V)$, with expansion (2.18). Then, for $i=1, \ldots, m$,

$$
G_{i} f: V \rightarrow B_{L}
$$

is defined by

$$
G_{i} f(x ; \theta)=\sum_{\mu \in M_{n}} Z_{L^{\prime}, L}\left(\partial_{i} f_{\mu}\right)(x) \theta^{\mu}
$$

Also, for $j=1, \ldots, n$,

$$
G_{j+m} f: V \rightarrow B_{L}
$$

is defined by

$$
G_{j+m} f\left(x_{j} \theta\right)=\sum_{\mu \in M_{n}} Z_{L^{\prime}, L}\left(f_{\mu}\right)(x) \theta^{\mu / j} \times(-1)^{\left|f_{\mu}(x)\right|},
$$

where $\left|f_{\mu}(x)\right|$ is the Grassmann parity of $f_{\mu}(x)$, and

$$
\theta^{\mu / j}=\theta^{\mu_{1}} \cdots \theta^{\mu_{i-1}} \theta^{\mu_{i+1}} \cdots \theta^{\mu_{k}}(-1)^{i-1}, \text { if } j=\mu_{i} \text { for some } i, 1 \leqq i \leqq k,
$$

and $\theta^{\mu / j}=0$ otherwise.

In the case of $G^{\infty}$ functions, odd derivatives are ambiguous because the value of $f$ is unaffected by the addition to $f_{\mu}$ of arbitrary multiples of $\beta_{1} \beta_{2} \cdots \beta_{L}$ (except when $\mu=\phi$ ). With higher derivatives the ambiguities become worse. With $G H^{\infty}$ functions the $f_{\mu}$ can only contain $\beta_{1}, \ldots, \beta_{L^{\prime}}$, and so ambiguities are no longer possible.

In [5] Sect. 2, various properties of $G^{\infty}(V)$ are proved; these are chain rules, product rules and so on, very much as for $C^{\infty}$ functions. These theorems also hold for $G H^{\infty}$ functions; in particular the Leibnitz rule holds, whereas for $G^{\infty}$ functions this is not quite true [9].

\section{Supermanifolds and the $L \rightarrow \infty$ Limit}

If one uses a finite number, $L$, of Grassmann generators, one can find cancellations occurring for the trivial reason that one runs out of new generators, and has repeated factors of generators inevitably occurring. The obvious intuitive idea is that if such cancellations threaten, one may avoid them by simply increasing $L$. This idea is explored in this section; $B_{L}^{m, n}$ is trivially extended, but if one has supermanifolds made by patching together pieces of $B_{L}^{m, n}$, care is needed to extend the patching properly. A method for extending a $G H^{\infty}$ supermanifold over a Grassmann algebra 
with $L$ generators to one over a Grassmann algebra with $L+1$ generators, and so on, is described in this section. This extension is a map between categories, and allows one to take the direct limit as $L$ increases. Batchelor has described the process for $H^{\infty}$ supermanifolds [8]; the present result is a straightforward adaption of her method to the slightly more complicated case of $G H^{\infty}$ supermanifolds. The starting point is the definition of a $G H^{\infty}$ supermanifold.

Definition 3.1. Let $Y$ be a Hausdorff topological space. (a) An $(m, n)$ chart on $Y$ over $B_{L}$ is a pair $(U, \psi)$ with $U$ an open subset of $Y$ and $\psi$ a homeomorphism of $U$ onto an open subset of $B_{L}^{m, n}$.

(b) An $(m, n) G H^{\infty}$ structure on $Y$ over $B_{L}$ is a collection $\left\{\left(U_{\alpha}, \psi_{\alpha}\right) \mid \alpha \in \Lambda\right\}$ of $(m, n)$

charts on $Y$ such that (i) $Y=\bigcup_{\alpha \in \Lambda} U_{\alpha}$, and (ii) for each pair $\alpha, \beta$ in $\Lambda$ the mapping $\psi_{\beta} \circ \psi_{\alpha}^{-1}$ is a $G H^{\infty}$ mapping of $\psi_{\alpha}\left(U_{\alpha} \cap U_{\beta}\right)$ onto $\psi_{\beta}\left(U_{\alpha} \cap U_{\beta}\right)$, (iii) the collection $\left\{\left(U_{\alpha}, \psi_{\alpha}\right) \mid \alpha \in \Lambda\right\}$ is a maximal collection of open charts for which (i) and (ii) hold. (c) An $(m, n)$ dimensional $G H^{\infty}$ supermanifold over $B_{L}$ is a Hausdorff topological space $Y$ with an $(m, n) G H^{\infty}$ structure over $B_{L}$.

This definition is a straightforward modification of the definition of $G^{\infty}$ supermanifold given in reference [5]; the reason for using $G H^{\infty}$ rather than $G^{\infty}$ functions is, as explained above, that this provides one with well defined odd derivatives. The various constructions and theorems described in Sect. 5 of [5] for $G^{\infty}$ supermanifolds can be repeated for $G H^{\infty}$ supermanifolds simply by replacing $G^{\infty}$ by $G H^{\infty}$ and $B_{L}$ module by $B_{L^{\prime}}$ module (where $L^{\prime}$ is the least integer not less than $L / 2$ ). For instance, one may define $G H^{\infty}$ functions on $G H^{\infty}$ supermanifolds; if $U$ is an open subset of a $G H^{\infty}$ supermanifold, let $G H^{\infty}(U)$ denote the set of $G H^{\infty}$ functions on $U$. Then $G H^{\infty}(U)$ is a $B_{L^{\prime}}$ module; also, if $U$ is a coordinate neighbourhood, the derivations of $G H^{\infty}(U)$ form a free $G H^{\infty}(U)$ module. (This result is not true for $G^{\infty}$ supermanifolds, as pointed out by Boyer and Gitler [10]; it is one of the principal motivations for replacing the $G^{\infty}$ concept by the more complicated $G H^{\infty}$ concept.)

Now, as it stands, the definition of $G H^{\infty}$ supermanifold is too general; the nilpotent pieces can be added on in various non-trivial ways [5], whereas any limiting process will require some unity in the way these things are added on. In principle there are many ways of refining the definition of supermanifold to achieve this. The way taken here will be that due to De Witt [4] which allows a correspondence between the sheaf theoretic and geometric approach to supermanifolds (see Sect. 4). The idea is to use a coarser topology on $B_{L}^{m, n}$ (called the De Witt topology). In this topology a subset $V$ of $B_{L}^{m, n}$ is open if and only if $V=\varepsilon_{m, n}^{L-1}(U)$ for some open subset $U$ of $\mathbb{R}^{m}$.

Definition 3.2. (Continuing Definition 3.1).

(d) The $G H^{\infty}$ supermanifold $Y$ is a $G H^{\infty}$ De Witt supermanifold $\left(D G H^{\infty} S M\right)$ if, for each $\alpha$ in $\Lambda$, the set $\psi_{\alpha}\left(U_{\alpha}\right)$ is open in $B_{L}^{m, n}$ in the De Witt topology.

The set $D G H^{\infty} S M(L)$ of $D G H^{\infty} S M$ 's over $B_{L}$ with odd dimension less than $\left[\frac{1}{2} L\right]$ is a category with morphisms maps $f: Y \rightarrow Z$ (where $Y, Z$ are members of $D G H^{\infty}$ $S M(L))$ which are $G H^{\infty}$ on coordinate patches.

It is now possible to describe the crucial step in the limiting process, the means by 
which a given $(m, n)$ dimensional $D G H^{\infty} S M$ over $B_{L}$ is extended to an $(m, n)$ dimensional $D G H^{\infty} S M$ over $B_{L+1}$.

Definition 3.3. Suppose that $L$ is a positive integer. Then the map $I_{L, L+1}: D G H^{\infty} S M(L) \rightarrow D G H^{\infty} S M(L+1)$ is defined in the following way. Suppose $Y \in D G H^{\infty} S M(L)$ with dimension $(m, n)$ and $G H^{\infty}$ structure as in Definitions 3.1 and 3.2. Now, for each $\alpha$ in $\Lambda$, let

and

$$
Z^{\alpha}=\varepsilon_{m, n}^{-1(L+1)} \varepsilon_{m, n}^{(L)} \quad\left(\psi_{\alpha}\left(V_{\alpha}\right)\right)
$$

Also, let

$$
Z=\bigcup_{\alpha \in \Gamma} Z^{\alpha}
$$

$$
Z^{\alpha ; \beta}:=\varepsilon_{m, n}^{-1(L+1)} \varepsilon_{m, n}^{(L)}\left(\psi_{\alpha}\left(V_{\alpha} \cap V_{\beta}\right)\right) .
$$

Then let $\tau_{\beta \alpha}:=Z^{\alpha ; \beta} \rightarrow Z^{\beta ; \alpha}$, with

$$
\tau_{\beta \alpha}=Z_{L, L+1}\left(\psi_{\beta} \circ \psi_{\alpha}^{-1}\right)
$$

(the map $Z_{L, L+1}$ is defined by Eq. 2.13). Define on $Z$ the relation $\sim$ with, given $p, q$ in $Z, p \sim q$ if and only if $p=\tau_{\alpha \beta} q$, where $\alpha, \beta$ are the unique elements of $Z$ such that $p \in Z_{\alpha}$ and $q \in Z_{\beta}$. Then it may be proved that $\sim$ is an equivalence relation, and that $Z / \sim$ is an $(m, n)$-dimensional $D G H^{\infty} S M$ over $B_{L+1}$.

$$
I_{L+1, L}(Y):=Z / \sim \text {. }
$$

An outline proof will now be given (following the same method that Batchelor used for $H^{\infty}$ supermanifolds [8]) that $I_{L+1, L}$ is an inclusion of categories, and that consequently a direct limit over $L$ can be taken.

Proposition 3.4. The category $D G H^{\infty} S M(L)$ includes as a full sub-category of $D G H^{\infty}$ $\operatorname{SM}(L+1)$.

Proof (outline). The inclusion $I_{L, L+1}$ has already been defined. Suppose $f: X \rightarrow Y$ is a morphism of $D G H^{\infty} S M$ over $B_{L}$. Then the correct morphism $f^{\prime}: I_{L, L+1}(X) \rightarrow$ $I_{L, L+1}(Y)$ may readily be defined using the mapping $Z_{L, L+1}$ on mappings on coordinate patches.

Definition 3.5. Let $D G H^{\infty} S M$ denote the direct limit of the categories $D G H^{\infty} S M(L)$.

The category $D G H^{\infty} S M$ may loosely be referred to as the category of $D G H^{\infty} S M$ 's over a Grassmann algebra with an infinite number of generators.

\section{The Sheaf Theoretic Approach}

In references [5] and [8] the correspondence between the graded manifold of Berezin and Leites [1] and Kostant [2] (which use the sheaf theoretic approach) and a certain class of $G^{\infty}$ supermanifold is demonstrated; the abstract algebras of the sheaf theoretic approach are realised as algebras of $H^{\infty}$ functions.

Suppose that $Y$ is a De Witt $H^{\infty}$ supermanifold over $B_{L}\left(D H^{\infty} S M\left(B_{L}\right)\right)$. (That is, $Y$ is as in Definitions 3.1 and 3.2 with $H^{\infty}$ everywhere replacing $G H^{\infty}$.) Then the 
following relation $\sim$ is an equivalence relation on $Y: p \sim q$ if and only if there exists $\alpha \in \Lambda$ such that both $p \in U_{\alpha}$ and $q \in U_{\alpha}$, and also

$$
\varepsilon_{L}^{m, n}\left(\psi_{\alpha}(p)\right)=\varepsilon_{L}^{m, n}\left(\psi_{\alpha}(q)\right)
$$

The fact that $\sim$ is an equivalence relation is proved in [8], together with the fact that $Y / \sim=B(Y)$ is an $m$-dimensional real $C^{\infty}$ manifold with $C^{\infty}$ structure $\left\{\left(V_{\alpha}, \phi_{\alpha}\right) \mid \alpha \in \Lambda\right\}$, where

$$
V_{\alpha}=\left\{\bar{p} \mid p \in U_{\alpha}\right\}
$$

and $\phi_{\alpha}: V_{\alpha} \rightarrow \mathbb{R}^{m}$ with

$$
\phi_{\alpha}(\bar{p})=\varepsilon_{L}^{m, n} \circ \psi_{\alpha}(\bar{p}) .
$$

Following De Witt [4], $B(Y)$ is called the body of $Y$. Now it can easily be shown that $H^{\infty}\left(U_{\alpha}\right)$ (the set of $H^{\infty}$ functions of $U_{\alpha}$ ) satisfies

$$
H^{\infty}\left(U_{\alpha}\right) \simeq C^{\infty}\left(V_{\alpha}\right) \otimes \Lambda\left(\mathbb{R}^{n}\right) .
$$

This follows almost immediately from the definition of $H^{\infty}$ (Eq. 2.17). From here it is a short step to show that $\left(B(Y), H^{\infty} \circ \varepsilon^{-1}\right)$ is a graded manifold. (Here $\varepsilon$ denotes projection from $Y$ onto $B(Y)$.) In [5] and [8] it is shown conversely that given any graded manifold $(X, A)$ there exists a supermanifold $Y$ such that $X=B(Y)$ and the sheaves $A$ and $H^{\infty} \circ \varepsilon^{-1}$ are isomorphic.

As remarked in the introduction, it is necessary for applications to supersymmetry to extend the sheaves in the graded manifold formalism, so that locally the graded algebras have the form (Eq. (1.4))

$$
A\left(U_{\alpha}\right) \simeq C^{\infty}\left(U_{\alpha}\right) \otimes \Lambda\left(\mathbb{R}^{n}\right) \otimes \Lambda\left(\mathbb{R}^{L^{\prime}}\right),
$$

where $L^{\prime}$ is some positive integer greater than $n$. These graded manifolds will be called graded manifolds of the extended kind. Suppose that $Y$ is now a $D G H^{\infty} S M$ (rather than $H^{\infty}$, as in the discussion above) with $G H^{\infty}$ structure $\left\{\left(U_{\alpha}, \psi_{\alpha}\right) \mid \alpha \in \Lambda\right\}$. Then the construction of $B(Y)$ can be repeated as for the $H^{\infty}$ case, and one has

$$
G H^{\infty}\left(U_{\alpha}\right) \simeq C^{\infty}\left(V_{\alpha}\right) \otimes \Lambda\left(\mathbb{R}^{n}\right) \otimes \Lambda\left(\mathbb{R}^{[L / 2]}\right) .
$$

Then the pair $\left(B(Y), G H^{\infty} \circ \varepsilon\right)$ is a graded manifold of the extended kind. Conversely, given an extended graded manifold $(X, A)$, a $D G H^{\infty} S M Y$ can be constructed so that $X=B(Y)$, and the sheaves $A$ and $G H^{\infty} \circ \varepsilon^{-1}$ are isomorphic. (Proofs are not given because they are trivial extensions of results in [5] and [8].)

Thus one has a complete correspondence between graded manifolds of the extended type and De Witt $G H^{\infty}$ supermanifolds. This correspondence could be stated formally as an equivalence of categories (as in [8]). It becomes a matter of taste whether one uses the graded manifold or supermanifold language; certainly the limiting process required to handle an infinite number of Grassmann generators becomes much simpler in the graded manifold approach (where one simply needs the embedding $l_{L^{\prime}, L}$ (Eq. 2.8) of a Grassmann algebra into one with more generators). On the other hand theoretical physicists, who are the main users of this kind of mathematics, will probably find supermanifold language more user friendly. 


\section{Summary}

In Sect. 2 the new class of functions on $B_{L}^{m, n}, G H^{\infty}$ functions, are suitable for superfields, but avoid the ambiguities in odd derivatives which have plagued other attempts. In Sect. $3 G H^{\infty}$ functions are used to define a new class of supermanifolds, which, benefiting from the better derivatives have nicely behaved vector fields. Also, now that the problem of defining a supermanifold over a finite-dimensional Grassmann algebra is satisfactorily solved, a new approach to the infinitedimensional case, via a direct limit, is possible. Section 4 shows the correspondence between extended graded manifolds and $G H^{\infty}$ supermanifolds.

To those physicists who use supermanifolds, but do not often lie awake at night worrying about the finer points of analysis, the message of this paper is simple-if you need more generators for your Grassmann algebra, help yourself?

\section{References}

1. Berezin, F. A., Leites, D. A.: Supervarieties. Sov. Math. Dokl. 16, 1218-1222 (1975)

2. Kostant, B.: Graded manifolds, graded Lie groups and prequantisation. Differential geometry in mathematical physics. Lecture Notes in Mathematics, Vol. 570, pp. 177-306. Berlin, Heidelberg, New York: Springer 1977

3. Volkov, D. V., Akulov, V. P.: Is the neutrino a Goldstone particle? Phys. Lett. 46B, 109-110 (1973); Abdus, S., Strathdee, J.: Super-gauge transformations, Nucl. Phys. B76, 477-482 (1974)

4. De Witt, B. S.: Supermanifolds. Cambridge: Cambridge University Press 1984

5. Rogers, A.: A global theory of supermanifolds. J. Math. Phys. 21, 1352-1365 (1980)

6. Batchelor, M.: Graded manifolds and supermanifolds. In: Mathematical aspects of superspace. Clarke, C. J. S., Rosenblum, A., Siefert, H. J. (eds.). Dordrecht: Reidel 1984

7. Jadzcyk, A., Pilch, K.: Superspace and supersymmetries. Commun. Math. Phys. 78, 373-390 (1981)

8. Batchelor, M.: Two approaches to supermanifolds. Trans. Am. Math. Soc. 258, 257-270 (1980)

9. Hess, H., Wieloch, M.: (private communication)

10. Boyer, C., Gitler, S.: The theory of $G^{\infty}$ supermanifolds. Trans. Am. Math. Soc. 285, 241-268 (1984)

11. Rothstein, M.: On the resolution of some difficulties in the theory of $G^{\infty}$ supermanifolds. Washington University preprint

Communicated by R. Haag

Received October 1, 1985; received again December 11, 1985 